\title{
Image Denoising using Nystrom Approximation with Glide Framework
}

\author{
K.Sri Hari Rao, P.Rohitha \\ ksrihariraoece@gmail.com \\ rohithapeddisetty@gmail.com
}

\begin{abstract}
Image denoising is an important image processing task, both as a process itself and as a component in other processes. Images are often corrupted by noise during the acquisition process. Denoising aims at eliminating this measurement noise while trying to preserve important signal features such as texture and edges. Over the past few decades, a large variety of algorithms has been developed for that purpose. Image denoising is an important process in image processing. In previous approach there are two denoising approach has been implemented such as NLM and Block-Matching and 3D (BM3D).In Non Local Means (NLM) each pixel has been estimated separately and fusing other "similar" neighborhood pixel. Whereas Block-Matching and 3D filtering (BM3D) is performed on group of similar patches together. In this paper, our proposed approach is a Global denoising algorithm (GLIDE) which is a non-local patch-based Processing where each pixel is estimated from all pixels in the image. Approximated spectral decomposition is computed using Nystrom Method. Eigen decomposition of the approximated image is estimated by Sinkhorn method for getting orthogonal eigenvectors of the obtained Eigen values with the help of Orthogonalization process. Finally Denoised image will be obtained using this proposed strategy. Hence, our approach can effectively enhance the performance of existing filters.
\end{abstract}

Keywords: Image denoising, Block- Matching and 3D (BM3D), NLM (Non-local means) filters, BM3D, Nystrom extension.

\section{INTRODUCTION}

Digital images play an important role both in daily life applications such as satellite television, magnetic resonance imaging, Computer tomography as well as in areas of research and technology such as geographical information systems and astronomy. A digital image is a numeric representation (normally binary) of a two-dimensional image. Depending on whether the image resolution is fixed, it may be of vector. Vector images resulted from mathematical geometry (vector). In mathematical terms, a vector consists of point that has both direction and length. Image viewer software displays images. Web browsers can display standard internet image formats including GIF, JPEG, and PNG. Digital Images are electronic snapshots taken of a scene or scanned from documents, such as photographs, manuscripts, printed texts, and artwork. The digital image is sampled and mapped as a grid of dots or picture elements (pixels). Each pixel is assigned a Tonal value (black, white, shades of gray or color), which is represented in binary code (zeros and ones). The binary digits ("bits") for each pixel are stored in a sequence by a computer and often reduced to a mathematical representation (compressed). The bits are then interpreted and read by the computer to produce an analog version for display or printing. Digital image processing deals with manipulation of digital images through a digital computer. It is a subfield of signals and systems but focus particularly on images. DIP focuses on developing a computer system that is able to perform processing on an image. The input of that system is a digital image and the system process that image using efficient algorithms, and gives an image as an output. Digital image processing enables the reversible, virtually noise-free modification of an image in the form of a matrix of integers instead of the classical darkroom manipulations or filtration of time-dependent voltages necessary for analog images and video signals. Even though many image processing algorithms are extremely powerful, the average user often applies operations to digital images without concern for the underlying principles behind these manipulations. The images that result from careless manipulation are often severely degraded or otherwise compromised with respect to those that could be produced if the power and versatility of the digital processing software were correctly utilized. Denoising of images is perhaps the most basic image restoration problem. The degradation model for the denoising problem can be described as: 


$$
\mathbf{y}=\mathbf{z}+\mathbf{e}
$$

Where column vectors $\mathbf{z}$ and $\mathbf{y}$ denote the (vectorized) underlying latent image and its noisy observation, respectively. The vector e represents zero-mean white noise1 with variance $\sigma 2$ (which is assumed to be spatially invariant in this paper). There have been numerous denoising algorithms to estimate $\mathbf{z}$ from $\mathbf{y}$, and in general most of these methods can be categorized as patch-based filters.

\section{LiTERATURE SURVEY}

Some Denoising approaches were processed in previous work such as the bilateral filter, LARK and NLM. These are estimated each pixel separately fusing other "similar" neighborhood pixels. Additional more recent state-of-the-art patch-based methods such as BM3D and PLOW denoised a group of similar patches together. Fig.1 shows the NLM kernel process.

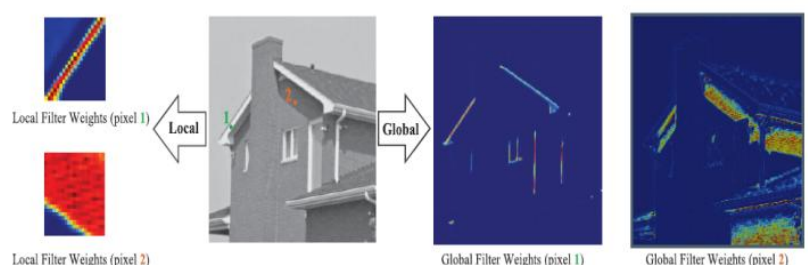

Fig. 1. Comparison of the local and global filter weights for the NLM kernel. The filter weights are computed for the two labeled pixels.

A bilateral filter is an edge-preserving and noise reducing smoothing filter. The intensity value at each pixel in an image is replaced by a weighted average of intensity values from nearby pixels. This weight can be based on a Gaussian distribution. LARK- Locally Adaptive Regression Kernels are very effective descriptors perform Denoising, Deblurring and Super-resolution without explicit priors. NLM(Non-local means) is an algorithm in image processing for image denoising. Unlike "local mean" filters, which take the mean value of a group of pixels surrounding a target pixel to smooth the image, non-local means filtering takes a mean of all pixels in the image. Block-Matching and 3D filtering (BM3D) is a grouping and collaborative filtering where noisy image is divided into blocks in a sliding manner. Each block is processed by searching similar blocks with fixed threshold. These matched blocks are stacked together to form a 3D array. Patch-based Locally Optimal Wiener Filtering (PLOW) for Image Denoising is needed to estimate the covariance matrix for the full color patches, whereby the dependencies across color channels can be captured implicitly. The filtering process is defined as applying a shrinkage function to the transform coefficients and recovering the estimated patches by inverse transform. However, Performance of these patch-based methods is strictly dependent on how well the similar patches are matched. For filters, locally adaptive regression kernel (LARK) denoising method and Non Local Means (NLM) measured the Eigenvectors and Eigen values which are well represented the image content. These methods robustly measuring the pixel similarity based on geodesic distance and point-wise photometric distance with patch distances. Again SAIF method is proposed where images are filtered local patch manner and iteration number are automatically optimized with locally estimated MSE.

\subsection{Disadvantage of Existing Systems}

- NLM filtering has been derived under the assumption of additive white Gaussian (AWG) noise contamination since NLM is known to fail at low values of SNR.

- In NLM used method PSNR and visual quality are required to improve.

- In NLM processing, Edges and Smooth features of an image are preserved but required much improvement.

- The SAIF algorithm not suited for theoretical limitation over this local window size, computational burden of building a matrix filter for a window as large as the whole image is prohibitively high.

- BM3D performs best when the number of matched blocks is higher (e.g., textures, regular shaped image structures, or uniform areas). It is not

- Effective when a large amount of matching blocks is not found. 
- In some cases the filtering process using BM3D is to remove some of the edge information making edges blurry.

- The adaptive choosing of matching distance in BM3D is useful for dealing with the noisy image with high noise level.

\section{Proposed System}

Our Proposed method can efficiently work with huge affinity (similarity) matrices by operating on only a small portion of the complete matrix to produce a low-rank approximation. Here new Nystrom method was introduced for finding numerical solutions to Eigen decomposition problems. This Nystrom extension used for much application such as manifold learning, image segmentation, and image editing. A key aspect of this Nystrom method is the distribution according to which columns are sampled from the original matrix. Combination of Nystrom approximation and Statistical Analysis gives an efficient solution, before processing to the filter approximation behavior of the filter MSE is analyzed. Obtained filter Eigen values are the shrinkage factors which directly tune the filtering performance. That shrinkage factors directly proportional to the signal-to-noise ratio of each channel. From the estimation of MSE, the shrinkage (k) and truncation (m) factors are calculated to extend the minimization problem. This shrinkage factors directly tune the filtering performance and Truncation factor increases the bias and lowers the variance. Also Iteration are estimated the filtering strength. Due to high computational and storage cost need eigen-decomposition of the original pixel which is approximated by Nystrom method. Next Sinkhorn method is applied to estimate the eigen decomposition of the symmetric. This Sinkhorn states that every square matrix with positive entries can be written in a certain standard form. To Orthogonal the approximated eigenvectors is done by Orthogonalization procedure. Orthogonalization is the process of finding a set of orthogonal vectors that span a particular subspace. Finally the Orthonormal approximation for eigen-decomposition is obtained.

\subsection{Advantages of Proposed System}

- Global filtering is inherently good in performance in a way that proposed methods are not.

- To denoised every single pixel our proposed global filtering uses all pixels of the input image.

- In terms of both PSNR \& Visual Quality the proposed global filtering algorithm performed well over the best existing patch-based methods.

- Performance brought by the global approach will grow substantially with increasing image size.

\subsection{Block Diagram}

Block diagram for proposed system.

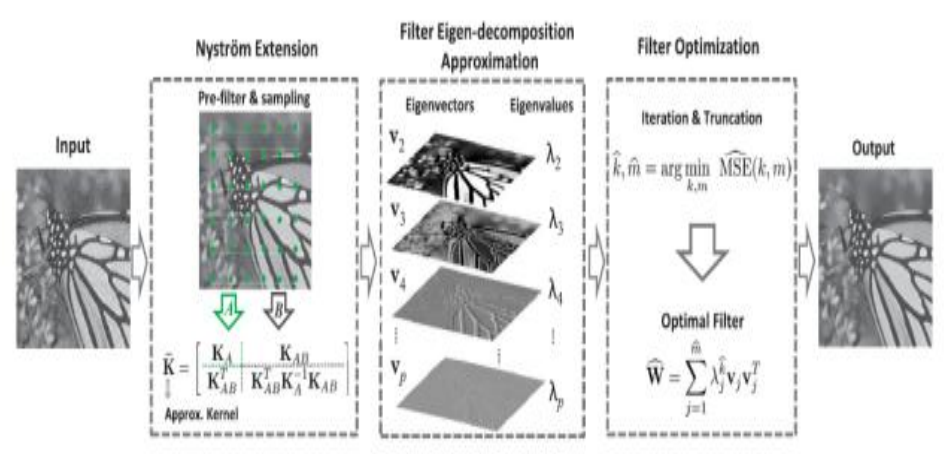

\section{ModuleS}

1. Pre Processing.

2. Nystrom Approximation.

3. Sinkhorn Method.

4. Orthogonalization. 


\section{Module Description}

\section{Pre Processing}

In image processing concept preprocessing process are required to evaluate further processing the image. In preprocessing steps we can resize the image with specified size. We added noise to the image in terms of sigma value. If increased sigma value added noise ratio is increased so that PSNR value gradually decreased. Here we going to perform denoising operation so there is a need to add the noise to image.

\section{Nystrom Approximation}

This method is a numerical approximation for estimating the eigenvectors of the symmetric kernel matrix K:

$$
\mathbf{K}=\Phi \Pi \Phi^{T}
$$

Where $\Phi=\left[\phi_{1}, \ldots\right.$, reppresents the Orthonormal eigenvectors and $\Pi=\left[\pi_{1}, \pi_{2}, \ldots, \pi_{n}\right]$ contains the Eigen values of $\mathbf{K}$. The Nystrom method seeks the numerical solution of an integral equation by replacing the integral with a representative weighted sum. The continuous problem is broken into $\mathrm{n}$ discrete intervals; quadrature or numerical integration determines the weights and locations of representative points for the integral. The discrete problem to be solved is now a system of linear equations. The Nystrom method is an efficient technique used to speed up large-scale learning

Algorithm 1: Spectral Approximation of the Filter W

Input: Sub-blocks of the similarity matrix $\mathbf{K}$ :

$\left\{\mathbf{K}_{A}, \mathbf{K}_{A B}\right\}$, let $(p, p)=\operatorname{size}\left(\mathbf{K}_{A}\right)$ and $(p, n-p)=\operatorname{size}\left(\mathbf{K}_{A B}\right)$

Output: $p$ leading orthogonal eigenvectors and eigenvalues of the approximated filter $\widetilde{\mathbf{W}}:\{\tilde{\mathbf{V}}, \widetilde{\mathbf{S}}\}$

Nyström Approximation:

1- $\mathbf{K}_{A}=\Phi_{A} \Pi_{A} \Phi_{A}^{T}$;

$\Rightarrow$ Eigen-decomposition of the sub-block $\mathbf{K}_{A}$

2- $\widetilde{\boldsymbol{\Phi}}=\left[\begin{array}{c}\boldsymbol{\Phi}_{A} \\ \mathbf{K}_{A B}^{T} \boldsymbol{\Phi}_{A} \Pi_{A}^{-1}\end{array}\right]$;

$\Rightarrow$ Approximate the $m$ leading eigenvectors of $\mathbf{K}$

Applications by generating low-rank approximations. Crucial to the performance of this technique is the assumption that a matrix can be well approximated by working exclusively with a subset of its columns. Spectral graph theoretic methods have recently shown great promise for the problem of image segmentation. However, due to the computational demands of these approaches, applications to large problems such as spatiotemporal data and high resolution imagery have been slow to appear. The computational demands can be solved for the numerical solution of Eigen function problems known as the Nystrom method.

$$
\int_{a}^{b} h(x) \mathrm{d} x \approx \sum_{k=1}^{n} w_{k} h\left(x_{k}\right)
$$

\section{Application of the Nystrom Method}

1. Spectral Clustering

2. Kernel Ridge Regression

3. Support Vector Machines

4. Kernel Logistic Regression

5. Manifold Learning 


\section{Sinkhorn Method}

Sinkhorn's theorem states that every square matrix with positive entries can be written in a certain standard form. Then the Sinkhorn-Knopp algorithm can be used to balance the matrix, that is, to find a diagonal scaling of $\$ A \$$ that is doubly stochastic. It is known that the convergence is linear, and an upper bound has been given for the rate of convergence for positive matrices. Sinkhorn proved that every entry-wise positive matrix can be made doubly stochastic by multiplying with two diagonal matrices.

Algorithm 2: Spectral Approximation of the Filter W

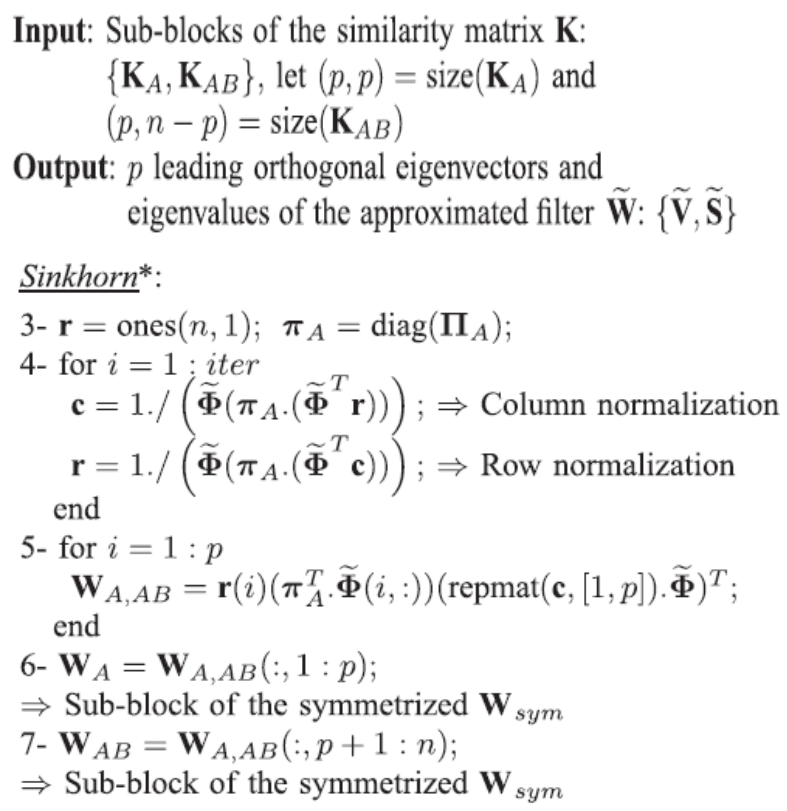

Here the Sinkhorn method is applied to estimate the Eigen decomposition of the symmetric and double-stochastic filter.

\section{Orthogonalization}

Orthogonalization is the process of finding a set of orthogonal vectors that span a particular subspace. Formally, starting with a linearly independent set of vectors in an inner product space (most commonly the Euclidean space Rn), orthogonalization results in a set of orthogonal vectors that generate the same subspace as the vectors. Every vector in the new set is orthogonal to every other vector in the new set; and the new set and the old set have the same linear span.

Algorithm 3: Spectral Approximation of the Filter W

Input: Sub-blocks of the similarity matrix $\mathbf{K}$ :

$$
\begin{aligned}
& \left\{\mathbf{K}_{A}, \mathbf{K}_{A B}\right\}, \text { let }(p, p)=\operatorname{size}\left(\mathbf{K}_{A}\right) \text { and } \\
& (p, n-p)=\operatorname{size}\left(\mathbf{K}_{A B}\right)
\end{aligned}
$$

Output: $p$ leading orthogonal eigenvectors and eigenvalues of the approximated filter $\widetilde{\mathbf{W}}:\{\widetilde{\mathbf{V}}, \widetilde{\mathbf{S}}\}$

\section{Orthogonalization:}

8- $\mathbf{W}_{A}^{1 / 2}=\operatorname{sqrtm}\left(\mathbf{W}_{A}\right)$;

9- $\mathbf{Q}=\mathbf{W}_{A}+\mathbf{W}_{A}^{-1 / 2} \mathbf{W}_{A B} \mathbf{W}_{A B}^{T} \mathbf{W}_{A}^{-1 / 2}$;

10- $\mathbf{Q}=\mathbf{V}_{Q} \mathbf{S}_{Q} \mathbf{V}_{Q}^{T}$

$\Rightarrow$ Eigen-decomposition of the symmetric matrix $\mathbf{Q}$

11- $\tilde{\mathbf{V}}=\left[\begin{array}{c}\mathbf{W}_{A} \\ \mathbf{W}_{A B}^{T}\end{array}\right] \mathbf{W}_{A}^{-1 / 2} \mathbf{V}_{Q} \mathbf{S}_{Q}^{-1 / 2}$

$\Rightarrow$ Approximated orthogonal eigenvectors

12- $\widetilde{\mathbf{S}}=\mathbf{S}_{Q} ; \Rightarrow$ Approximated eigenvalues 


\section{RESUlts}

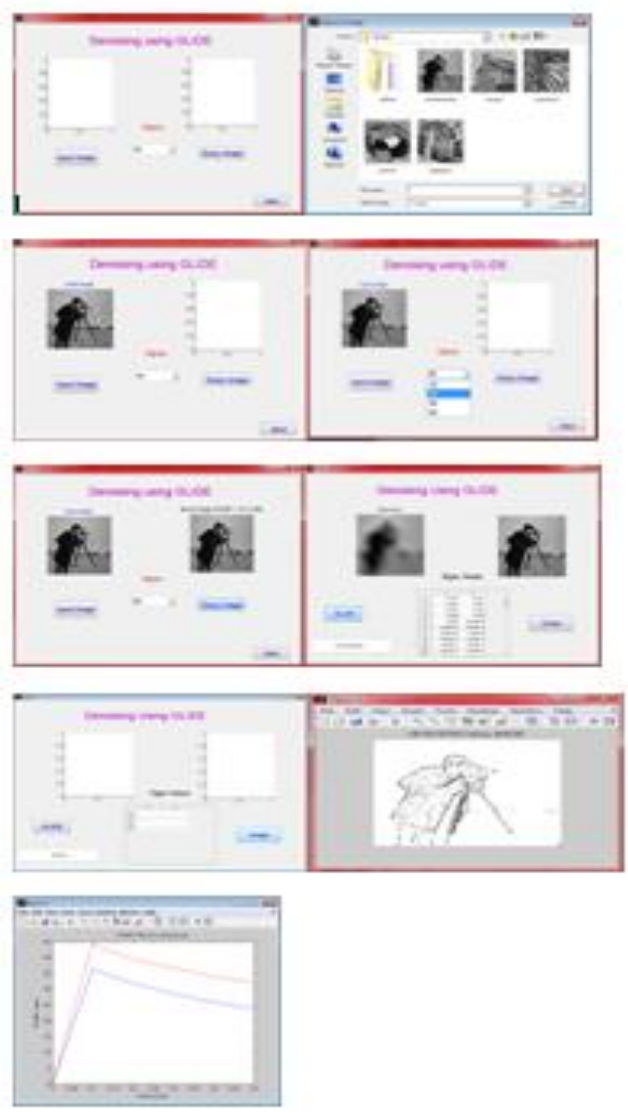

\section{CONCLUSION}

Global denoising image has been implemented which is a truly global denoising algorithm. The global approach goes beyond the dominant paradigm of non-local patch-based processing, which we have shown here to be inherently limited. At the same time, the experimental results demonstrated that the proposed approach improves over the best existing patch-based methods in terms of both PSNR and subjective visual quality. More specifically, the PSNR values for the global filter point to essentially perfect reconstruction of the noise-free image, which is apparently impossible to achieve of algorithms like such as NLM, BM3D, even if all the filter parameters are known exactly. Hence, our denoising algorithm (GLIDE) which is a non-local patch-based processing where each pixel is estimated from all pixels in the image. Compute the approximated spectral decomposition using Nystrom Method. Estimate the Eigen decomposition of the approximated image by Sinkhorn method for getting orthogonal eigenvectors of the obtained Eigen values with the help of Orthogonalization process. Finally Denoised image will be obtain using this proposed strategy. Hence, our approach can effectively enhance the performance of existing filters.

\section{REFERENCES}

[1]. C. Tomasi and R. Manduchi, "Bilateral filtering for gray and color images," in Proc. 6th ICCV, Jan. 1998, pp. 836-846.

[2]. H. Takeda, S. Farsiu, and P. Milanfar, "Kernel regression for image processing and reconstruction," IEEE Trans. Image Process., vol. 16, no. 2, pp. 349-366, Feb. 2007.

[3]. A. Buades, B. Coll, and J. M. Morel, "A review of image denoising algorithms, with a new one," Multiscale Model. Simul., vol. 4, no. 2, pp. 490-530, 2005.

[4]. K. Dabov, A. Foi, V. Katkovnik, and K. Egiazarian, "Image denoising by sparse 3-D transformdomain collaborative filtering," IEEE Trans. Image Process., vol. 16, no. 8, pp. 2080- 095, Aug. 2007. 
[5]. P. Chatterjee and P. Milanfar, "Patch-based near-optimal image denoising," IEEE Trans. Image Process., vol. 21, no. 4, pp. 1635-1649, Apr. 2012.

[6]. P. Milanfar, "A tour of modern image filtering: New insights and methods, both practical and theoretical," IEEE Signal Process. Mag., vol. 30, no. 1, pp. 106-128, Jun. 2013.

[7]. L. Zhang, W. Dong, D. Zhang, and G. Shi, "Two-stage image denoising by principal component analysis with local pixel grouping," Pattern Recognit., vol. 43, pp. 1531-1549, Apr. 2010.

[8]. M. Elad and M. Aharon, "Image denoising via sparse and redundant representations over learned dictionaries," IEEE Trans. Image Process., vol. 15, no. 12, pp. 3736-3745, Dec. 2006.

[9]. P. Chatterjee and P. Milanfar, "Is denoising dead?" IEEE Trans. Image Process., vol. 19, no. 4, pp. 895-911, Apr. 2010.

[10]. A. Levin, B. Nadler, F. Durand, and W. T. Freeman, "Patch complexity, finite pixel correlations and optimal denoising," in Proc. ECCV, Oct. 2012, pp. 73-86.

[11].H. Talebi, X. Zhu, and P. Milanfar, "How to SAIF-ly boost denoising performance," IEEE Trans. Image Process., vol. 22, no. 4, pp. 1470-1485, Apr. 2013.

[12].C. Williams and M. Seeger, "Using the Nyström method to speed up kernel machines," in Proc. $A d v$. NIPS, 2001, pp. 682-688.

[13].E. Nyström, "Über die Praktische Auflösung von linearn ingtegraglechungen mit anwendungen auf Randwertaufgaben der potentialtheorie," Commentationes Phys. Math., vol. 4, no. 15, pp. 152, Apr. 1928.

[14].C. T. Baker, The Numerical Treatment of Integral Equations. Oxford, U.K.: Clarendon Press, 1977.

[15]. A. Talwalkar, S. Kumar, and H. Rowley, "Large-scale manifold learning," in Proc. IEEE Conf. CVPR, Jun. 2008, pp. 1-8. 